\title{
Modification of a method of Galanopoulos for determining earthquake risk
}

\author{
R. MAAZ (*) \\ Ricevuto il 20 Maggio 1969
}

\begin{abstract}
Summary. - A method for the determination of earthquake risk basing on the energy-frequency-relation proposed by Galanopoulos is modified.
\end{abstract}

Riassunto. - Nella nota viene modificato il metodo per la determinazione del rischio legato ad un terremoto, metodo già proposto precedentemente da A. G. Galanopoulos e basato sulla relazione che intercorre tra energia e frequenza.

In the relation

$$
\lg N=a-b M
$$

between earthquake magnitude $M$ and annual number $N$ of earthquakes of the magnitude class with centre $M$ the coefficient a appears to depend mainly on the energy release in the considered region $\Gamma$ with area $G$, whereas $b$ depends on the mean depth of the focus in $I$ '. $\triangle$ fter reducing [1] to a general standard area $G^{*}$ the accordingly transformed relation [1] informs us about the relative frequency of such earthquakes. Supposing all earthquakes have equal depth, then a measure of the earthquake risk would be found by this. To compensate the actual variation of the mean focal depth and the variability of $b$ commected with it Galanopoulos (1) proceeds as follows. May

$$
\lg N^{\prime}=a_{0}-b_{0} M
$$

(*) Institut Physik der Erde, Bereich I (Geodynanik und Physik des Erdimern). Jena (DDR). 
substitutes [1] where $b_{0}$ is a fixed reasonable value, possibly 0.8 . The formulas [1] and [2] are connected by postulating that the magnitude $M_{0}$ corresponds to a certain value $N_{0}$ in both cases

$$
M=M_{0} \longrightarrow N^{\prime}\left(M_{0}\right)=N\left(M_{0}\right)=N_{0} .
$$

Therefore from [1] and [2] follows

$$
a_{0}=a \frac{b_{0}}{b}+\left(1-\frac{b_{0}}{b}\right) \lg N_{0} .
$$

In the next step Galanopoulos ( $\left.{ }^{1}\right)$ gets to the frequency $N^{\prime *}$ related to the standard area by thinking the earthquake events being distributed homogenously in $\Gamma$ and $\Gamma^{*} \subset \Gamma$, too. That means

$$
\frac{N^{*}}{N^{\prime}}=\frac{G^{*}}{G}
$$

whence

$$
\lg N^{*} *=a^{*}-b_{0} M, \quad a^{*}=a_{0}+\lg \frac{G^{*}}{G}
$$

Now we consider two regions $\Gamma_{1}$ and $\Gamma_{2}$, the intersection of which need not be empty, with $a_{1} \neq a_{2}$, but having equal parameters $a^{*}, b$

$$
a^{\prime *}{ }_{1}=a^{\prime *_{2}}, \quad b_{1}=b_{2} \neq b_{0} .
$$

For such regions it holds naturally

$$
\frac{N_{1}}{N_{2}}=\frac{G_{1}}{G_{r_{2}}} .
$$

[6] helps with [5], [4], [7], and [1] to

$$
0=a^{\prime *}{ }_{1}-a^{\prime} *_{2}=\left(1-\frac{b_{0}}{b_{1}}\right) \lg \frac{N_{01}}{G_{1}} \frac{G_{2}}{N_{02}} .
$$

Therefore

$$
\frac{N_{01}}{G_{1}}=\frac{N_{02}}{G_{2}}-n_{0}
$$

is a quantity which must be chosen uniquely for all regions $\Gamma$ and $N_{0}$ cannot be independent of $\Gamma$. With this condition we get

$$
a^{\prime *}=\frac{b_{\mathrm{f}}}{b}\left(a-\lg n_{0} G\right)+\lg n_{0} G^{*}
$$

as modified measure of earthquake risk in the sense of Galanopoulos. 
The last term is an arbitrary number independent of $a$, $b$ and $a$, suitably defined to zero:

$$
n_{0} r^{*}=1
$$

Therefore we dillally get:

$$
a^{* *}=\frac{b_{0}}{b}\left(: \quad \lg \frac{G}{G^{*}}\right) .
$$

The values of $b_{0}$ and $a^{*}$ must be chosen definitly and according to reality, possibly in conformity with Galanoponlos as

$$
b_{0}=0.8, \quad G^{*}=10^{1} \mathrm{~km}^{2} .
$$

The arbitariness in choosing $b_{0}$, $r^{*}$ and $\psi_{0}(r *$ can be comprehended as a foible of the method. Another difficulty is that the investigated region $I$ 'annot be defined without free choice. Moreover, there remains the open question mentioned also by Galanoponlos to which degree the basing relation [1] fits to reality.

Furthermore, we consider the difference between $a^{*}$ and the analogous quantity after Galanopoulos who generally postulates

$$
N_{0}=H_{0}(r=1,
$$

instead of [8] where $V_{0}$ is defined in [3] and who, therefore, gets

$$
a^{*}=\frac{b_{0}}{l} a+\lg \frac{a^{*}}{\frac{a}{a}}
$$

The difference

$$
a^{\prime *}-a^{*}=\left(1-\frac{b_{0}^{\prime}}{b}\right) \lg \frac{G^{\prime}}{G^{*}}
$$

to [9] depends on the chosen values $b_{0}$ and $G^{*}$ and of comse on $b$ and (r. In Tab. I in the paper of Galanoponlos, this difference does not exceed the amomnt of 0.11 in the ane of South Califormia where $a=5.25, b=0.86, \quad G=29.61 G^{*}$, and $a^{*}=3.41, a^{\prime *}=3.52$.

Finally, we can see at a consequence of [10] that this postulate is msuitable. Let $I_{1}$ and $I_{2}^{\prime}$ be two regions with equal relative quantities ${ }^{*}$ and $b, b$ different from $b_{0}$ :

$$
a_{1}^{*}=a_{2}^{*}, \quad b_{1}=b_{2} \neq b_{1} .
$$


This leads with [11] and [1] to

$$
\frac{N_{1}}{N_{2}}=\left(\frac{G_{1}}{G_{2}}\right)^{b_{1} / b_{0}}
$$

contradictory to the natural condition [7] because of $b_{1} \neq b_{0}$.

Communications of the Zentralinstitut Physik der Erde, No 34.

\section{REFERENCES}

(1) Galanopoulos A. G., On Quantitative Determination of Earthquake Risk.

"Annali di Geofisica", XXI, 2, 193-206, (1968). 